\title{
Bacterial isolation and antibiotic susceptibility from diabetic foot ulcers in Kenya using microbiological tests and comparison with RT-PCR in detection of S. aureus and MRSA
}

Daniel M. Mutonga ${ }^{1,6^{*}}$, Marianne W. Mureithi ${ }^{2,3}$, Nancy N. Ngugi ${ }^{4}$ and Fredrick C. F. Otieno ${ }^{5}$

\begin{abstract}
Objectives: Diabetic foot ulcers (DFUs) often lead to hospital admissions, amputations and deaths; however, there is no up-to-date information on microbial isolates from DFUs and no mention of utilization of molecular techniques in Sub-Saharan Africa. We conducted a cross-sectional study among 83 adult patients at a tertiary hospital in Kenya over 12 months. The study aimed to isolate, identify bacteria, their antibiotic susceptibility patterns in active DFUs, and to compare standard microbiological methods versus a real-time PCR commercial kit in the detection of Staphylococcus aureus DNA and methicillin-resistant S. aureus (MRSA) DNA.

Results: Eighty swabs (94\%) were culture-positive; 29\% were Gram-positive and 65\% were Gram-negative. The main organisms isolated were S. aureus (16\%), Escherichia coli (15\%), Proteus mirabilis (11\%), Klebsiella pneumoniae (7\%) and Pseudomonas aeruginosa (7\%). The bacterial isolates showed resistance to commonly used antibiotics such as ampicillin, amoxicillin, cefepime, ceftazidime, cefuroxime, clindamycin, erythromycin, piperacillin-tazobactam, tetracycline and trimethoprim-sulphamethoxazole (TMPSMX). Thirty-one percent of the S. aureus isolated and 40\% of the Gramnegatives were multi-drug resistant organisms (MDROs). There was a high prevalence of nosocomial bacteria. MRSA were not identified using culture methods but were identified using PCR. PCR was more sensitive but less specific than culture-based methods to identify S. aureus.
\end{abstract}

Keywords: Diabetic foot ulcers, Kenya, Multi-drug resistant organisms, Methicillin-resistant S. aureus, Polymerase chain reaction

\section{Introduction}

It is estimated that $10-15 \%$ of diabetic patients will develop diabetic foot ulcers (DFUs) at some point in their life $[1,2]$. In Africa, the overall prevalence of DFUs was found to be $13 \%$ in a recent meta-analysis [3]. At presentation, about half of DFUs are clinically infected [4]. Staphylococcus aureus and beta-haemolytic Streptococci are the most common causes of skin infections [5-11]. In resource-poor countries however, Gram-negatives like Pseudomonas aeruginosa are more prevalent $[6,10]$. In

\footnotetext{
*Correspondence: danielmutonga@gmail.com

${ }^{1}$ Institute of Tropical and Infectious Diseases, College of Health Sciences,

University of Nairobi, P.O Box 19676-00202, Nairobi, Kenya

Full list of author information is available at the end of the article
}

Kenya, S. aureus and Escherichia coli were found to be the most common organisms in DFIs [12]. More recently, $73.2 \%$ of DFUs were infected while $26.8 \%$ were culturenegative [13]. Fungal infections may also cause DFIs [14].

Antimicrobial resistance (AMR) is an emerging problem globally. Methicillin-resistant S. aureus (MRSA) was first observed in the early 1960s and has been associated with increased hospital stay, healthcare costs and mortality [15]. MRSA represented $4.7 \%$ of $S$. aureus isolated in a study in Morocco [16]. In Brazil, 33\% cases of MRSA (cefoxitin-resistant) were vancomycin-resistant [17]. Multi-drug resistant organisms (MDROs) are bacteria that are resistant to more than one or more classes of antibiotics. In Tanzania, antibiotic susceptibility tests 
(AST) of bacterial isolates from DFUs revealed a high AMR [18].

Polymerase chain reaction (PCR) is a molecular method that can be used to identify bacterial species by amplifying the $16 \mathrm{~S}$ ribosomal RNA (rRNA) gene $[4,10]$. Real-time PCR (RT-PCR) allows detection of DNA or RNA through production of fluorescence light during the reaction. In Sub-Saharan Africa, there is a lack of upto-date information on microbial isolates from diabetic foot ulcers and no mention of utilization of molecular techniques. The only available study from Africa is from Algeria where sequencing target genes identified a high prevalence of Gram-negative bacilli (54.9\%) and MDROs (58.5\%) [19].

The objective of this present study was therefore to isolate bacteria and determine their antibiotic susceptibility patterns in patients with infected DFUs using culturebased methods and to compare the differences between microbiological methods and RT-PCR in detecting $S$. aureus and MRSA in a sub-Saharan setting, which is facing an escalating AMR with extensive health, economic and societal implications.

\section{Main text \\ Methods \\ Study design and subjects}

This cross-sectional study was conducted at the Kenyatta National Hospital (KNH), Nairobi, Kenya-a national referral and teaching hospital. Eighty-three adult diabetic patients with any type of diabetes and having active foot ulcers were recruited by consecutive sampling from September 2017 to August 2018. Active foot ulcers were defined as non-healed ulcers during physical examination and were thought to be more likely infected.

\section{Microbiological methods}

After rinsing the wound area with normal saline, samples were collected using sterile cotton swabs from the centre of the diabetic wound and taken to the KNH Microbiology Laboratory immediately or within $2 \mathrm{~h}$. On Day 1 , specimens were inoculated using the streak method on Sheep Blood Agar and CLED Media and incubated under aerobic conditions at $35-37{ }^{\circ} \mathrm{C}$ for $24-48$ h. On Day 2, growth was noted as colonies on the culture media and the most predominant colony isolated using standard microbiological and biochemical tests. The VITEK ${ }^{\circledR} 2$ machine (bioMe'rieux, Durham, United States) was then utilized for further identification and AST.

\section{Screening for S. aureus and MRSA DNA using RT-PCR}

All specimens were stored at $-20{ }^{\circ} \mathrm{C}$ to $-80{ }^{\circ} \mathrm{C}$ for subsequent DNA isolation and PCR analysis at Biozeq Kenya Molecular Laboratory (based at KAVI-Institute of Clinical Research, University of Nairobi). Fifty-one samples were randomly selected from the 83 recruited patients and dissolved in $200 \mu \mathrm{L}$ to $500 \mu \mathrm{L}$ of Dulbecco's phosphate buffered solution (Sigma ${ }^{\circledR}$-Aldrich, Steinheim, Germany). Automated DNA extraction was performed using QIASymphony Kit (Qiagen ${ }^{\circledR}$, Hilden, Germany) according to manufacturer's instructions. PCR-amplification and real-time hybridization were conducted using the MRSA Quant Real-TM kit (Sacace ${ }^{\mathrm{TM}}$ Biotechnologies, Como, Italy). Amplification was set up in a $1.0 \mu \mathrm{L}$ PCR tubes containing $15 \mu \mathrm{L}$ of PCR Master Mix (PCR mix-1 FRT MRSA, PCR-mix-2 FRT, TaqF polymerase, and Internal Control). The reaction tubes were subjected to Thermal cycling reactions on a Rotor-Gene $\mathrm{Q}$ machine (Qiagen, Hilden, Germany) comprising of $15 \mathrm{~min}$ at $95^{\circ} \mathrm{C}$ followed by 5 cycles of $15 \mathrm{~s}$ at $95^{\circ} \mathrm{C}, 30 \mathrm{~s}$ at $60{ }^{\circ} \mathrm{C}$, and $15 \mathrm{~s}$ at $72{ }^{\circ} \mathrm{C}$, and finally 40 cycles of $15 \mathrm{~s}$ at $95^{\circ} \mathrm{C}, 30 \mathrm{~s}$ at $55^{\circ} \mathrm{C}$ and $15 \mathrm{~s}$ at $72{ }^{\circ} \mathrm{C}$ for measuring fluorescent signal.

\section{Quality control}

Positive controls for microbiological tests were recently collected ( $<7$ days) positive specimens of bacteria from human samples that were stored at room temperature in cotton swabs in a safety cabinet. For the RT-PCR tests, quality control was assured by running 4 additional samples alongside the 51 specimen: Positive control, Negative control, DNA Quality Standard (QS) 1 MRSA and DNA QS2 MRSA.

\section{Statistical analyses}

Microsoft Excel was used for data entry and data analysis. Data was represented as frequencies, percentages, tables and charts. Comparison of microbiological and RT-PCR was based on absolute numbers, sensitivity, specificity, positive predictive value (PPV), and negative predictive value (NPV). Both culture and real-time PCR were considered as the gold standard.

\section{Results \\ Bacterial isolates and antimicrobial sensitivity tests}

The most predominant growth on the culture plate per specimen was isolated. Out of 85 culture and susceptibility tests performed from 83 patients, 78 swabs had mono-microbial growth, 1 had poly-microbial growth (2 isolates) and 5 had no growth. Most organisms (64.71\%, $\mathrm{n}=55)$ were Gram-negative and $(29.41 \%, \mathrm{n}=25)$ organisms were Gram-positive. The most common organisms isolated were $S$. aureus $(16.47 \%, \mathrm{n}=14)$, E. coli $(15.29 \%$, $\mathrm{n}=13)$, Proteus mirabilis $(10.59 \%, \mathrm{n}=9)$, Klebsiella pneumoniae $(7.06 \%, \mathrm{n}=6)$ and $P$. aeruginosa $(7.06 \%, \mathrm{n}=6)$ (see Additional file 1: Figure S1). Some of the other rare bacteria isolated from patients included Staphylococcus lentus, Staphylococcus simulans, Staphylococcus xylosus, 
Acinetobacter baumannii, Burkholderia cepacia, Kocuria kristinae, Leuconostoc mesenteroides, Pantoea agglomerans, Providencia stuartii and Raoultella ornithinolytica.

Staphylococcus aureus was highly resistant to benzylpenicillin and trimethoprim-sulphamethoxazole (TMPSMX) but sensitive to cefoxitin, oxacillin, nitrofurantoin, levofloxacin, linezolid, and vancomycin. There was no MRSA identified using microbiology tests. Isolates of S. epidermidis, S. intermedius and S. simulans were either $100 \%$ resistant, $50 \%$ resistant or $100 \%$ sensitive to the antibiotics tested (Additional file 2: Table S1). E. coli was highly resistant to ampicillin, aztreonam, cefuroxime and TMPSMX but sensitive to amikacin and nitrofurantoin (refer to Additional file 2: Table S2). P. mirabilis showed a similar resistance to ampicillin but sensitivity to amikacin. $P$. aeruginosa was sensitive to ampicillin, amoxicillin-clavulanic acid, aztreonam, ceftazidime, ciprofloxacin, nitrofurantoin and TMPSMX. $S$. fonticola species showed resistance to ampicillin, amoxicillin, cefazolin, cefepime, ceftazidime, piperacillin-tazobactam and TMPSMX. About a third $(30.77 \%, \mathrm{n}=4)$ of the $S$. aureus were MDROs while $40.38 \%(n=21)$ Gramnegative bacilli were MDROs (Fig. 1).

\section{Culture-versus-molecular tests}

Molecular tests were performed on 51 out of the total 85 samples tested (Fig. 2). The RT-PCR results of the 51 samples were compared with the respective culture results for the detection of $S$. aureus and MRSA. For the Gram-positive pathogens, 11 were culture-positive for $S$. aureus while 7 yielded other Staphylococcus sp. RT-PCR for $S$. aureus was positive for 9 out of the 11 culture-positive results (see Additional file 2: Table S3). Five samples positive for S. aureus but negative for MRSA on culturebased methods were positive for MRSA on RT-PCR. Two samples positive for other Staphylococcus sp. and negative for S. aureus on culture were positive for MRSA on RT-PCR. One sample with other Staphylococcus sp. suspected to be skin contaminants based on microbiological tests underwent RT-PCR. The sample was negative for $S$. aureus on culture tests but positive for $S$. aureus and MRSA DNA on RT-PCR.

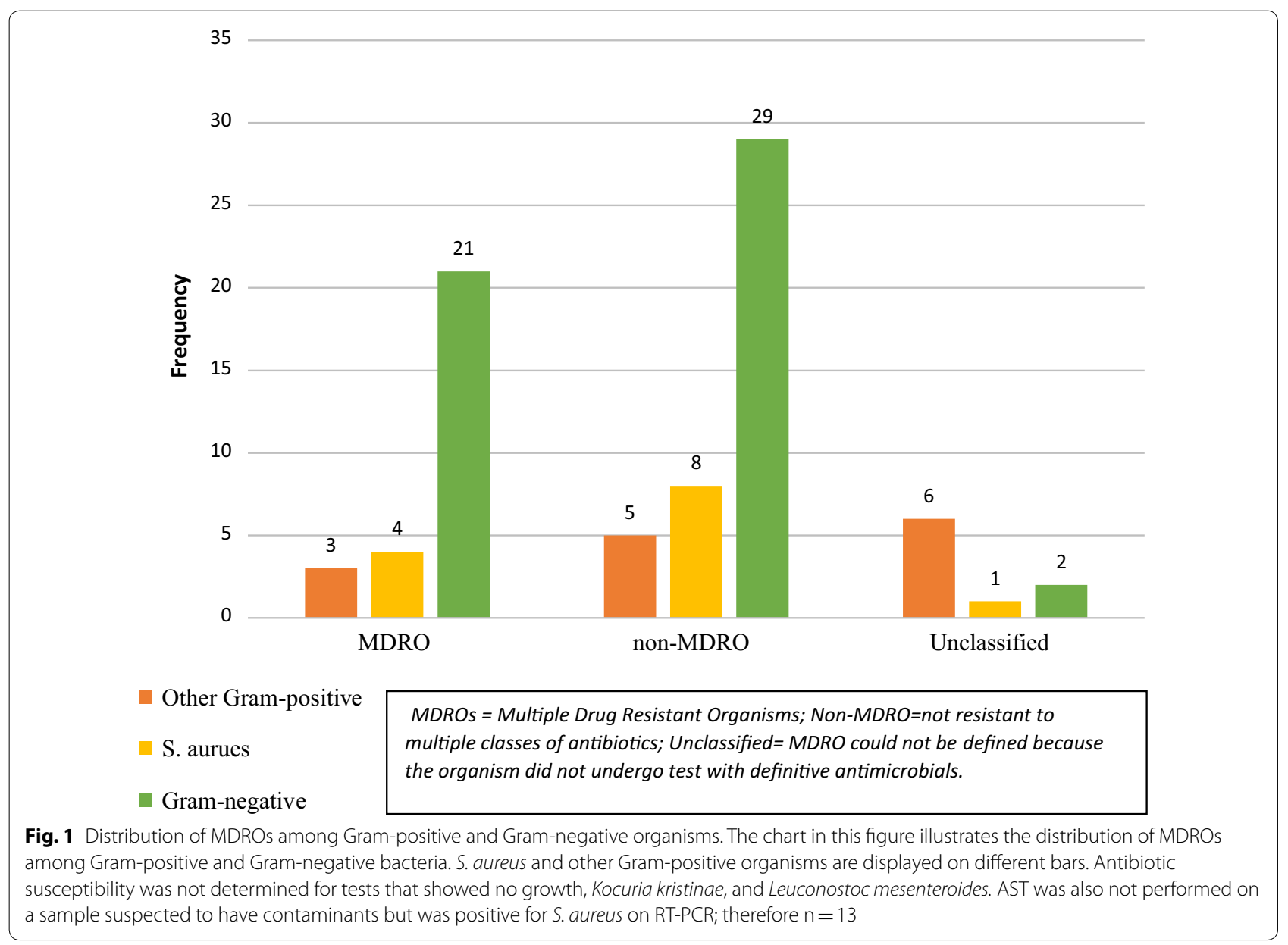



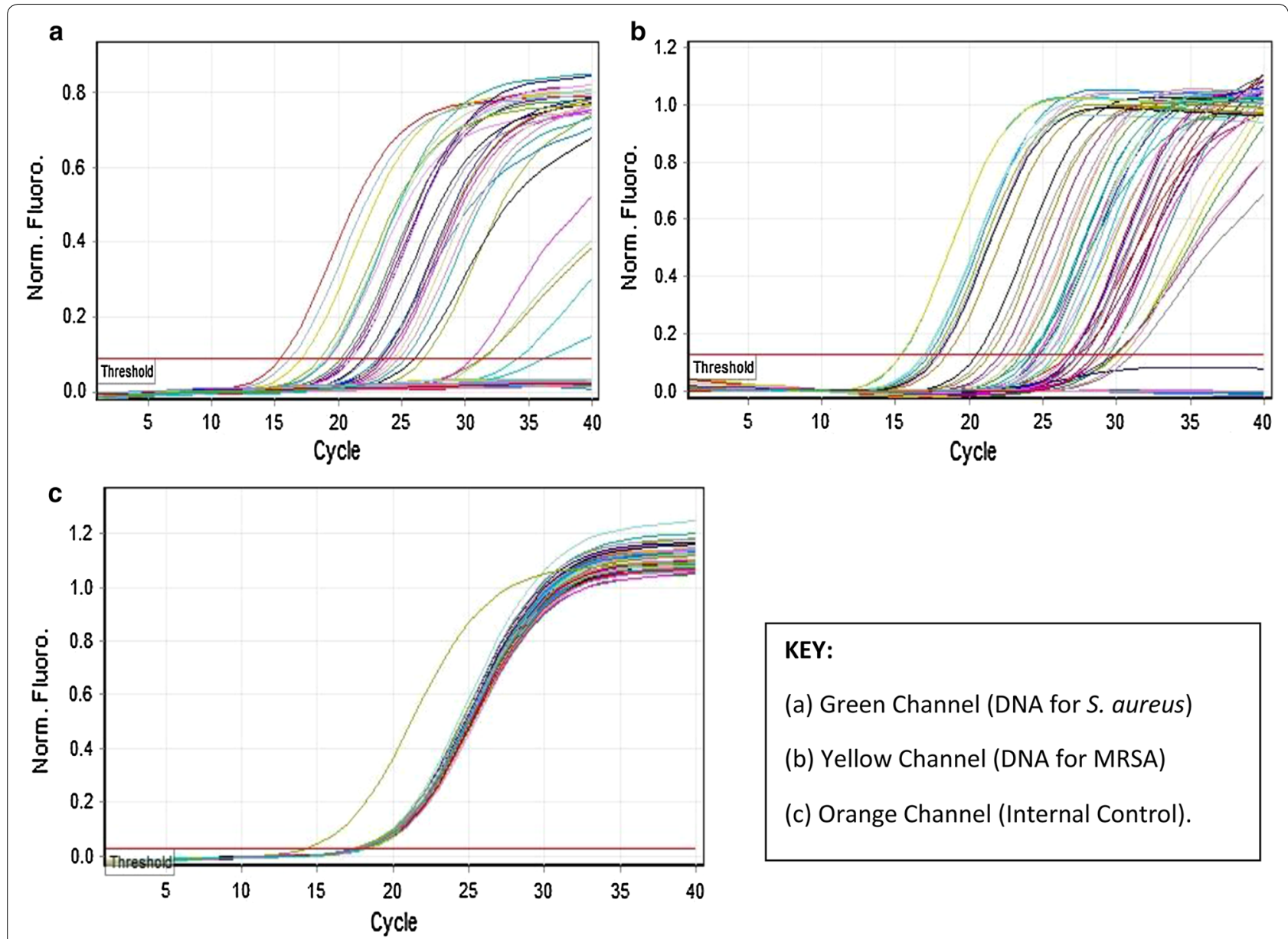

KEY:

(a) Green Channel (DNA for S. aureus)

(b) Yellow Channel (DNA for MRSA)

(c) Orange Channel (Internal Control).

Fig. 2 Quantitation data for Cycling A (PCR Reports). As shown in this figure, the $C_{t}$ used was $\geq 20$ since the exponential of the sigmoid curve of the Internal Control (Orange Channel) begins at this point

One sample without any growth was subjected to RT-PCR. The sample was positive for $S$. aureus DNA but not MRSA DNA. Four (12.5\%) samples that had Gram-negative bacteria based on culture tests were also positive for S. aureus on RT-PCR while 6 (18.8\%) samples from these batch were positive for MRSA DNA (Additional file 2: Table S4). Of importance is that MRSA were not identified using culture methods but were identified using PCR. There was amplification for MRSA in one sample that was culture positive for $S$. aureus but negative during RT-PCR for this pathogen (Additional file 2: Table S3). Atypical amplification for MRSA occurred in four samples that were both culture and RT-PCR negative for S. aureus (Additional file 2: Table S4).

Further comparison was then made by statistical analyses. Firstly, culture methods were considered the assay and RT-PCR as the reference. The sensitivity of the

Table 1 Comparison of RT-PCR and culture-based methods of S. aureus and MRSA in DFUs

\begin{tabular}{|c|c|c|c|c|c|c|}
\hline Species & Reference & Assay & Sensitivity (\%) & Specificity (\%) & PPV (\%) & NPV (\%) \\
\hline \multirow[t]{2}{*}{ S. aureus } & Culture & RT-PCR & 90.9 & 82.5 & 58.8 & 97 \\
\hline & RT-PCR & Culture & 58.8 & 97 & 90.9 & 66.7 \\
\hline \multirow[t]{2}{*}{ MRSA } & Culture & RT-PCR & & 72.7 & & 100 \\
\hline & RT-PCR & Culture & & 100 & & 72.7 \\
\hline
\end{tabular}

In this Table, both culture-methods and RT-PCR were used as gold standards. In the column heading, the gold standard is the "Reference" while the method being tested, the "Assay" 
VITEK $^{\circledR} 2$ machine to detect $S$. aureus was $90.9 \%$ while the specificity was $82.5 \%$ (Table 1 and Additional file 2: Table S5). The PPV was 58.8\% while the NPV was $97.0 \%$. The sensitivity and PPV of the culture tests to detect MRSA could not be calculated due to missing culturepositive results (Additional file 2: Table S5). However, its specificity was $72.7 \%$ and NPV was $100 \%$. In the second case, the RT-PCR was considered the assay and culture tests as the reference. The sensitivity, specificity, PPV and NPV of the RT-PCR to detect $S$. aureus were $58.8 \%$, $97.0 \%, 90.9 \%$ and $66.7 \%$ respectively (Table 1 ). For detection of MRSA, the specificity for the RT-PCR was $100 \%$ while the NPV was $72.7 \%$.

\section{Discussion}

DFU is a chronic issue that contributes significantly to morbidity and mortality. In this study, over $90 \%$ of the DFUs were infected. This was higher than in earlier studies in Kenya, Tanzania, and Libya where approximately $70 \%$ of DFUs had positive cultures $[13,18,20]$. S. aureus was the most predominant single species isolated in DFUs as reported in other studies [2, 6-10, 12, 17, 18, 20]. In this current study, Gram-negative bacteria were more predominant than Gram-positive organisms similar to studies in Morocco and Brazil [16, 17]. Similar to previous studies, $E$. coli and $P$. aeruginosa were common in this study $[6,10,12,17,20]$.

In this study, there was high AMR among the Gramnegative organisms compared to the Gram-positive bacteria. In fact, MDROs mainly consisted of Gram-negative bacteria. $S$. aureus was sensitive to most antibiotics including vancomycin whereas no MRSA was identified by culture methods (cefoxitin screen). From previous studies, MRSA is predominant in DFUs and shows limited AMR [21, 22]. In Brazil, 22\% of DFUs had MRSA following cefoxitin screen, and $33 \%$ of these were also resistant to vancomycin [17]. S. aureus and E. coli isolated from DFUs were classified as MDROs in this earlier study. From previous studies, antibiotics that used to work before are now showing increasing resistance [12, 18].

Biofilms, present in chronic wounds, are a defensive mechanism for bacteria against the effects of antibiotics and can explain the rise in AMR $[10,11]$. Unjustified use of antibiotics is another cause of AMR, misuse of health resources and a burden to patients and their families [6, 23, 24]. From this present study, amikacin is effective against most Gram-negative bacteria. The high AMR to ampicillin should warrant care during empirical treatment of DFUs in this setting [25]. Further, although some $E$. coli isolates were resistant to meropenem (a third-line antibiotic); all were sensitive to nitrofurantoin (a firstline antibiotic). There is therefore need to use antibiotics judiciously and be guided by routine culture and susceptibility tests.

However, more accurate tests should be explored since culture-based methods have been reported to have a high number of false-negatives [8]. In the present study, molecular tests were more sensitive but less specific than culture-based methods. PCR revealed pathogens that had not been recognized by culture-methods such as MRSA species. Previous research reveals a higher specificity for culture tests when compared to RT-PCR as a reference while a lower sensitivity, a slightly higher PPV and a higher NPV [8]. Similar to this earlier study, RT-PCR revealed more $S$. aureus than identified through culturemethods [8]. PCR is therefore an effective way of species identification in patients with DFUs.

\section{Limitations}

- Due to limited funding, only the most predominant organism was isolated from the samples. Further, anaerobic bacteria were also not identified.

- PCR technology may amplify dormant or dead bacteria in a sample.

- The study fails to explain the atypical amplification why RT-PCR is positive for MRSA but negative for $S$. aureus. Contamination or cross-reactivity is not a possibility since the RT-PCR kit should detect both $S$. aureus DNA and mecA gene specific for MRSA.

\section{Additional files}

Additional file 1: Figure S1. Distribution of Gram-positive and Gramnegative bacteria isolated.

Additional file 2: Table S1. Resistance patterns for Gram-positive organisms; Table S2. Resistance patterns for Gram-negative organisms; Table S3. Comparison of microbiological and molecular tests for Grampositive bacteria; Table S4. Comparison of microbiological and molecular tests for Gram-negative bacteria; Table S5. Distribution of organisms based on culture and RT-PCR results for S. aureus and MRSA.

Abbreviations

AMR: antimicrobial resistance; AST: antibiotic susceptibility tests; DFUs: diabetic foot ulcers; KNH: Kenyatta National Hospital; MDROs: multi-drug resistant organisms; MRSA: methicillin-resistant S. Aureus; NPV: negative predictive value; PCR: polymerase chain reaction; PPV: positive predictive value; RT-PCR: real-time PCR; TMPSMX: trimethoprim-sulphamethoxazole.

Authors' contributions

DMM — study design, literature search, data collection, data analysis, manuscript writing, editing and submission of the manuscript. MWM, NNN and FCFO also participated in study design, data analysis, manuscript writing \& editing. All authors read and approved the final manuscript. 


\begin{abstract}
Author details
${ }^{1}$ Institute of Tropical and Infectious Diseases, College of Health Sciences, University of Nairobi, P.O Box 19676-00202, Nairobi, Kenya. ${ }^{2}$ KAVI-Institute of Clinical Research, P.O Box 19676-00202, Nairobi, Kenya. ${ }^{3}$ Department of Medical Microbiology, College of Health Sciences, University of Nairobi, P.O Box 19676-00202, Nairobi, Kenya. ${ }^{4}$ Department of Medicine, Kenyatta National Hospital, P.O. Box 20723-00202 Nairobi, Kenya. ${ }^{5}$ Department of Clinical Medicine and Therapeutics, College of Health Sciences, University of Nairobi, P.O Box 19676-00202, Nairobi, Kenya. ${ }^{6}$ P.O Box 11692-00100, Nairobi, Kenya.
\end{abstract}

\section{Acknowledgements}

We would like to acknowledge all the patients and their relatives without whom this study would not have been possible. We are most grateful to the nursing staff in the Department of Medicine and the Diabetes and Endocrinology Outpatient Clinic at Kenyatta National Hospital for their support. Our appreciation also goes to the laboratory staff at KNH Microbiology Laboratory and Biozeq Kenya Laboratory for running the tests.

\section{Competing interests}

The authors declare that they have no competing interests.

\section{Availability of data and materials}

All data generated or analysed during this study is included in this published article and supplementary information files. Additional datasets are publicly available on the University of Nairobi Library Research Archive Website (http:// erepository.uonbi.ac.ke:8080/xmlui/handle/11295/105662) as part of the Master's Thesis of the corresponding author in Tropical and Infectious Diseases, 2018.

\section{Consent for publication}

Not applicable.

\section{Ethics approval and consent to participate}

Ethical approval was sought from the KNH Ethics and Research Committee (Approval Number P769/10/2016; Website: http://erc.uonbi.ac.ke/) and KNH Department of Medicine (Registration Number: Med/101/2017). Patients were enrolled into the study only after written informed consent was taken.

\section{Funding}

DMM received partial funding from KNH Research and Programs Department (Registration Number: Med/101/2017) to conduct this study. Website: http:// $\mathrm{knh}$.or.ke/. The funders had no role in study design, data collection and analysis, decision to publish or preparation of the manuscript.

\section{Publisher's Note}

Springer Nature remains neutral with regard to jurisdictional claims in published maps and institutional affiliations.

Received: 9 April 2019 Accepted: 23 April 2019

Published online: 29 April 2019

\section{References}

1. Boulton AJ. The diabetic foot: a global view. Diabetes Metab Res Rev. 2000;16(Suppl 1):S2-5.

2. Agwu E, Dafiewhare EO, Ekanem PE. Possible diabetic-foot complications in Sub-Saharan Africa. Glob Perspect Diabet Foot Ulcerations. 2010;2007:3-15.

3. Rigato M, Pizzol D, Tiago A, Putoto G, Avogaro A, Fadini GP. Characteristics, prevalence, and outcomes of diabetic foot ulcers in Africa. A systemic review and meta-analysis. Diabetes Res Clin Pract. 2018;142:63-73. https ://doi.org/10.1016/j.diabres.2018.05.016.

4. Sotto A, Lavigne JP, Dunyach-remy C, Lipsky BA. New molecular techniques to study the skin microbiota of diabetic foot ulcers. Adv Wound Care. 2015;4(1):38-49. https://doi.org/10.1089/wound.2014.0532.
5. Mendes JJ, Neves J. Diabetic foot infections: current diagnosis and treatment. J Diabet Foot Complicat. 2012;4(2):26-45.

6. Uckay I, Gariani I, Pataky Z, et al. Diabetic foot infections: state-of-the-art. Diabetes Obes Metab. 2014;16(4):305-16.

7. Lipsky BA, Richard JL, Lavigne JP. Diabetic foot ulcer microbiome: one small step for molecular microbiology one giant leap for understanding diabetic foot ulcers. Diabetes. 2013;62(3):679-81.

8. Stappers MHT, Hagen F, Reimnitz P, Mouton JW, Meis JF, Gyssens IC. Direct molecular versus culture-based assessment of Gram-positive cocci in biopsies of patients with major abscesses and diabetic foot infections. Eur J Clin Microbiol Infect Dis. 2015;34(9):1885-92.

9. Messad N, Prajsnar TK, Lina G, O'Callaghan D, Foster SJ, Renshaw SA, et al. Existence of a colonizing Staphylococcus aureus strain isolated in diabetic foot ulcers. Diabetes. 2015;64:2991-5.

10. Spichler A, Hurwitz BL, Armstrong DG, Lipsky BA. Microbiology of diabetic foot infections: from Louis Pasteur to "crime scene investigation". BMC Med. 2015;13:2. https://doi.org/10.1186/s12916-014-0232-0.

11. Banu A, Noorul Hassan MM, Rajkumar J, Srinivasa S. Spectrum of bacteria associated with diabetic foot ulcer and biofilm formation: a prospective study. Aust Med J. 2015;8(9):280-5.

12. McLigeyo, Otieno LS. Diabetic ulcers-a clinical and bacteriological study. East Afr Med J. 1991;68:204-10.

13. Nyamu PN, Otieno CF, Amayo EO, Mcligeyo SO. Risk factors and prevalence of diabetic foot ulcers At Kenyatta National Hospital, Nairobi. East Africa Med J. 2003;80(1):36-43.

14. Gitau AM, Ng'ang'a ZW, Sigilai W, et al. Fungal infections among diabetic foot ulcer-patients attending diabetic clinic in Kenyatta National Hospital, Kenya. East Afr Med J. 2011;88(1):9-17.

15. Ornskov D, Kolmos B, Horn PB, Nederby Nielsen J, Brandslund I, Schouenborg P. Screening for methicillin-resistant Staphylococcus aureus in clinical swabs using a high-throughput real-time PCR-based method. Clin Microbiol Infect. 2008;14(1):22-8. https://doi.org/10.111 1/j.1469-0691.2007.01880.x.

16. Belefquih B, Frikh M, Benlahlou Y, et al. Diabetic foot infection in morocco: microbiological profile. Wounds. 2016;28(3):89-98.

17. Perim MC, Borges J da C, Celeste SRC, Orsolin EDF, Mendes RR, Mendes $\mathrm{GO}$, et al. Aerobic bacterial profile and antibiotic resistance in patients with diabetic foot infections. Rev Soc Bras Med Trop. 2015;48(5):546-54. https://doi.org/10.1590/0037-8682-0146-2015.

18. Chalya PL, Mabula JB, Dass RM, Kabangila R, Jaka H, McHembe MD, et al. Surgical management of diabetic foot ulcers: a Tanzanian University Teaching Hospital Experience. BMC Res Notes. 2011;4(365):1-7. https:// doi.org/10.1186/1756-0500-4-365.

19. Djahmi N, Messad N, Nedjai S, Moussaoui A, Mazouz D, Richard JL, et al. Molecular epidemiology of Staphylococcus aureus strains isolated from inpatients with infected diabetic foot ulcers in an Algerian University Hospital. Clin Microbiol Infect. 2013;19(9):E398-404. https://doi. org/10.1111/1469-0691.12199.

20. Benkhadoura M, Alswehly M, Elbarsha A. Clinical profile and surgical management of diabetic foot in Benghazi. Libya. Foot Ankle Surg. 2016;22(1):55-8. https://doi.org/10.1016/j.fas.2015.04.011.

21. Chhibber S, Kaur TKS. Co-therapy using lytic bacteriophage and linezolid: effective treatment in eliminating methicillin resistant Staphylococcus aureus (MRSA) from diabetic foot infections. PLoS ONE. 2013;8(2):e56022.

22. Mathangi T, Prabhakaran P, Rayappan F, Tilton F. Isolation, molecular characterization and antibiogram of bacteria isolated from diabetic foot ulcers. Int J Curr Res Aca Rev. 2013;1 (1):17-25.

23. Reiber GE, Pecoraro RE, Koepsell TD. Risk factors for amputation in patients with diabetes mellitus. A case-control study. Ann Intern Med. 1992;117:97-105

24. Karugu L. Mainstreaming diabetic foot education in Kenya: prevalence of diabetes in Kenya. In: World, I. D. F., \& Congress D, editor. 2011.

25. Achieng L, Menge TOE. The KNH guide to empiric antibiotic therapy. 2nd ed. Nairobi: Kenyatta National Hospital; 2018. 